\section{Empirical Virtual Sliding Target Guidance Law Design: An Aerodynamic Approach}

\author{
P. A. RAJU \\ DEBASISH GHOSE, Member, IEEE \\ Indian Institute of Science
}

We present a novel empirical virtual sliding target (VST) guidance law for the midcourse phase of a long range surface-to-air missile that uses the simplicity of the conventional proportional navigation (PN) guidance law while exploiting the aerodynamic characteristics of a missile's flight through the atmosphere to enable the missile to achieve superior performance than that achieved by conventional PN guidance laws. The missile trajectory emulates the trajectory of an optimal control based guidance law formulated on a realistic aerodynamic model of the missile-target engagement. The trajectory of the missile is controlled by controlling the speed of a virtual target that slides towards a predicted intercept point during the midcourse phase. Several sliding schemes, both linear and nonlinear, are proposed and the effect of the variation of the sliding parameters, which control the sliding speed of the virtual target, on the missile performance, are examined through extensive simulations that take into account the atmospheric characteristics as well as limitations on the missile in terms of the energy available and lateral acceleration limits. Launch envelopes for these sliding schemes for approaching and receding targets are also obtained. These results amply demonstrate the superiority of the proposed guidance law over the conventional PN law.

Manuscript received March 29, 2001; revised October 8, 2002; released for publication August 13, 2003.

IEEE Log No. T-AES/39/4/822049.

Refereeing of this contribution was handled by J. L. Leva.

Authors' current addresses: P. A. Raju, TCS-Nortel Technology Lab, Parkwest-II, Raheja Estate, Kulupwadi Road, Borivali (E), Mumbai 400 066, India; D. Ghose, Dept. of Aerospace Engineering, Indian Institute of Science, Bangalore 560 012, India, E-mail: (dghose@aero.iisc.ernet.in).

0018-9251/03/\$17.00 (c) 2003 IEEE

\section{INTRODUCTION}

In the missile guidance literature on analytical design and performance evaluation of guidance laws, the equations of motion are generally restricted only to the kinematics of the engagement (for example, see [1-4]). Usually, the missile and target are represented as point objects moving with constant speeds and subject to guidance commands in the form of lateral accelerations which may or may not have a fixed bound. The analytical and numerical results on the performance of such guidance laws are based solely on kinematic equations that are not meant to represent the practical aspects of the motion of a flight vehicle through the atmosphere. One such aspect is the variation in the aerodynamic forces to which a surface-to-air-missile is subjected to as it flies from almost sea level to very high altitudes. Another aspect is related to the missile speed which undergoes drastic variations that depends on the atmospheric characteristics, the missile's aerodynamic properties, and the missile propulsion system's thrust profile, among several other factors. Further, the missile lateral acceleration (latax) is constrained by a bound that depends on the dynamic pressure and which changes with the altitude.

For performance evaluation of a guidance law on a realistic missile, these factors are normally taken into account in elaborate six-degree-of-freedom simulations. However, there is another aspect to this problem that needs to be addressed. Empirical guidance laws-such as the proportional navigation (PN) class of guidance laws-are designed based on certain intuitive concepts and are not always optimal. To get the best performance out of a missile it is natural to look at the optimality aspect of guidance laws leading naturally to optimal control formulations (for example, see [5, 6]). Although the analysis from these optimal control formulations give excellent insight into the optimality of guidance laws, the performance of these guidance laws are only valid in an ideal scenario. Further, even with simplified equations of motion, the solution of the optimal control problem turns out to be quite difficult. When more realistic models are considered, obtaining a feasible optimal solution becomes virtually impossible.

This situation was to some extent alleviated by the singular perturbation (SP) approach $[7,8]$, where realistic models were used to formulate the optimal control problem and solved in an approximate sense by time-scale separation of state variables. Physical properties of missiles, target, atmospheric characteristics, and engagement scenarios are explicitly considered in the formulation to maximize the accuracy of guidance law performance evaluation. However, it was necessary to make several assumptions in order to render the SP approach 
feasible. These strong assumptions naturally detract from the optimality of the guidance law. Also, one of the major problems that SP approach faces is that of identification of the time-scale structure of systems having nonlinear dynamics. Only recently some satisfactory results in this direction that uses Lyapunov exponents and direction fields to characterize time-scales have become available [9-11]. However, it must be said that even with strong assumptions and a simplified approach to time-scale separation, the SP approach showed a distinct advantage over the earlier optimal control formulations in the sense that it took into account the aerodynamic behavior of the missile and the typical characteristics of the atmosphere to produce a guidance law that effectively exploited these factors. A paper that uses a realistic model while following the conventional optimal control approach where the solution is obtained by extensive numerical iteration to solve the resultant two-point-boundary-value-problem (TPBVP) is [12]. However, the large computational burden makes this guidance law impossible to be implemented in real-time.

However, one of the major results that emerges from these papers $[7,8,12]$ is that the missile must initially pitch-up and rise to some high altitude in order to conserve its energy and to gain higher speed as it finally pitches down to intercept the target. Although this maneuver does not improve the missile's miss-distance performance to any significant extent, the major returns are in terms of a larger launch envelope due to the missile's higher forward speed at the end of the midcourse phase. Another point that emerges from these studies $[7,8,12]$ is the inherent computational burden of these guidance laws and their dependence on uncertain aerodynamic parameters. Indeed the conventional PN law scores over these guidance laws in terms of simplicity, robustness, and elegance.

We attempt here to merge the simplicity of the PN law with the intuitive understanding of the improved launch envelope performance of the SP guidance law, with the ultimate objective of formulating an empirical virtual sliding target (VST) guidance law. The proposed empirical guidance law is easy to implement since it uses the well-known PN law as its basic guidance scheme and uses a significant feature of the SP guidance law to improve performance of the missile. Simulations of several trajectories and corresponding launch envelopes for approaching and receding targets reveal the superiority of this law when compared with conventional guidance laws. We do not claim any optimality for the proposed guidance law, except that which accrues logically from the usage of PN and the exploitation of the aerodynamic characteristics of the missile. Moreover, the VST guidance law is used only in the midcourse phase, as in $[8,12]$, and hence its launch boundary performance is the only aspect considered here. Performance in terms of miss-distances was not addressed here since a miss-distance analysis is more relevant to the evaluation of the terminal phase guidance. Another point worth noting is that the word "sliding" used here should not be construed to mean that this scheme has any relation to the "sliding mode control" techniques. In fact, the word sliding refers to the behavior of the synthetically defined virtual target, which will be explained shortly.

\section{BASIC CONCEPTS}

\section{A. Guidance Phases}

Before discussing the structure of the VST guidance law we explain the different guidance phases of the missile, which are important in designing the guidance law. For any surface-to-air missile there are three guidance phases. The first part of the trajectory is called the boost phase, which occurs for a very short time. At the completion of this phase, midcourse guidance is initiated. The function of the midcourse guidance phase is to place the missile at a point such that the target is within the terminal acquisition range of its seeker with the missile seeker pointed in an appropriate direction with respect to the target. The last few seconds of the engagement constitutes the terminal guidance phase, which is a most crucial phase since its success or failure determines the success or failure of the entire mission. In the terminal phase, the missile locks on to the target and attempts to close the distance to the target as quickly as possible under the constraints of fuel and maneuver limitations. In the boost phase, the thrust is very high and it is necessary to use this initial thrust as efficiently as possible, so that we may get better guidance performance.

\section{B. Singular Perturbation Guidance Law}

A solution technique based on time-scale separation forms the cornerstone of the SP guidance law $[7,8]$. Without going into the mathematical details of time scale separation and the resulting simplified optimal control problems, we will attempt an intuitive understanding of the physics behind the SP guidance law. Essentially, the SP guidance law exploits the fact that at higher altitudes the low atmospheric density results in a low drag on the missile. Because of this, the initial high thrust during the boost phase imparts a higher speed to the missile than would be possible with a conventional guidance law which ignores the high altitude speed advantages and commands the missile to fly at an altitude (which could be low initially) prescribed by the guidance law based on its sole consideration of achieving interception. If 


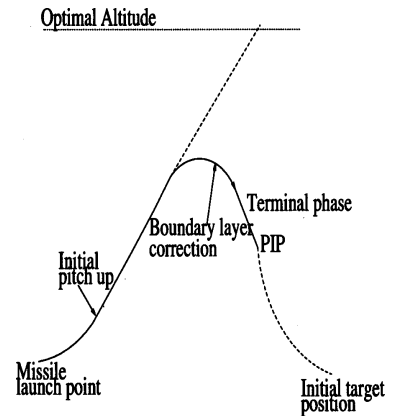

Fig. 1. Trajectory for a SP-based guidance law.

the initial altitude of the missile due to this guidance law is low then the missile has to overcome high drag initially and consequently lose velocity. This is especially important from the viewpoint that the higher the missile velocity at the end of the midcourse phase, the better is the chance of interception and higher is the launch envelope boundary. For instance, for a surface-to-air missile, or even for an air-to-air missile, when the target is at a higher altitude than the missile launch point, according to the SP guidance law the missile initially pitches up in a vertical maneuver plane defined by the current missile position (which is initially the missile launch point) and the predicted intercept point (PIP). This pitch-up maneuver may be preceded by another maneuver which aligns the missile velocity vector with the vertical maneuver plane and makes the in-plane pitch-up maneuver possible. When we consider a planar engagement scenario, this initial maneuver is not necessary and the missile executes the pitch-up maneuver directly.

The extent of the pitch-up maneuver is determined by an optimal altitude $h^{*}$ whose exact value is computed in the slowest time-scale formulation that optimizes the drag on the missile by balancing the effect of varying altitude and missile velocity on the drag force given the average thrust on the missile. The missile aims for a point directly above the PIP at a height equal to this optimal altitude. But this is not sufficient to achieve an intercept since to do so successfully the missile has to actually move towards the PIP. So, after a certain period of time (decided by the engagement considerations) a boundary layer correction is performed that forces the missile to pitch down gradually and finally point towards the PIP (see Fig. 1). This boundary layer correction and its initiation is fairly arbitrary and is decided upon by the guidance law designer [8].

The computational burden imposed by the SP guidance law is less than a standard optimal control formulation, but it is nevertheless almost twice as large as that required by PN [13]. Moreover, the computations are based on some very strong and arbitrary assumptions. Our attempt in the next section is to retain the essential idea of the initial

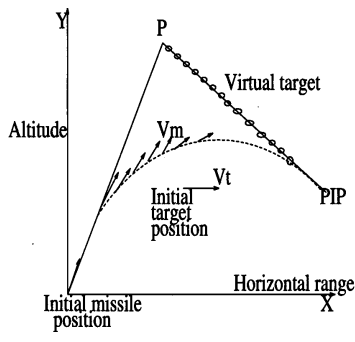

Fig. 2. VST.

pitch-up maneuver of the missile and use it to design an empirical guidance law that emulates the SP guidance law in its trajectory characteristics, is simple to implement, and gives superior performance to conventional PN guidance laws.

\section{VIRTUAL SLIDING TARGET GUIDANCE LAW}

\section{A. Virtual Target}

As in the SP-based guidance law, in the VST guidance law also we initially allow the missile to guide itself towards a stationary point at a high altitude. However, we select this stationary point based on the initial geometry of the engagement without going through the process of iteratively solving for the optimal altitude as is done in the SP approach. In the three-dimensional scenario the PIP and the missile position would play a role in determining the plane on which this stationary point is located, and depending on the change in the PIP and the missile position, as the engagement progresses, the orientation of this plane would also change. However, we restrict ourselves to engagements on a vertical plane here. Thus, the PIP, the missile position, and the stationary point will all remain on this vertical plane throughout the engagement.

This stationary point is now considered as a virtual target. This is shown in Fig. 2 as the point $P$. As shown in Fig. 2, the missile initially ignores the actual target and guides itself towards the stationary virtual target by pitching up during the initial high thrust phase. At the end of the high thrust boost phase, the guidance commands are generated by the missile guidance system based on the assumption that the virtual target moves towards the PIP with some speed. We may consider the virtual target as a bead sliding on a string connecting the point $P$ and the PIP. The sliding speed of the virtual target and its variation with time are important guidance design considerations since by controlling the sliding speed we can control the trajectory of the missile, the altitude that the missile achieves, and its terminal velocity. Ideally, the sliding speed of the virtual target should satisfy the boundary condition that the virtual target reaches the PIP at the same time as the missile. This is, however, not of vital importance since the 


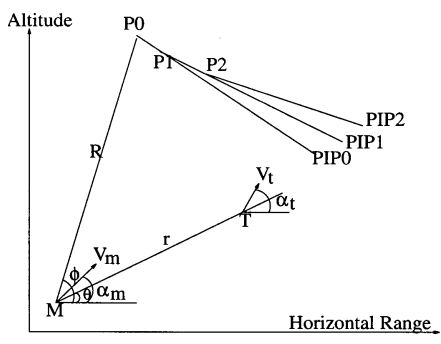

Fig. 3. Motion of VST.

artifice of using a virtual target is only resorted to during the midcourse phase and the final terminal engagement is taken care of by the homing guidance phase. Thus, in each guidance cycle the position of the virtual target and the PIP is updated. Note that a guidance cycle is defined as a fixed interval of time, at the beginning of which the guidance command is computed and is kept constant during the whole of this interval. Thus, it is also defined as the periodic interval at which the successive computation of the guidance command is done. We describe the computation of the position of the virtual target and the PIP in the next section.

The above idea is explained with the help of Fig. 3 as follows. A typical engagement scenario is assumed in which the missile and target start from arbitrary points in space with some initial velocities. The initial position of the virtual target is denoted as $P_{0}$. The virtual target moves along the line connecting $P_{0}$ and the initial PIP (denoted by PIP0) with some velocity. Then position of the virtual target after one guidance cycle is denoted by $P_{1}$. In the next guidance cycle the missile guides itself towards $P_{1}$, during which period the virtual target moves along the line joining $P_{1}$ and the freshly computed PIP (denoted by PIP1). The resultant virtual target position at the end of this guidance cycle is denoted by $P_{2}$. The missile guides itself towards $P_{2}$ during the next guidance cycle. This process continues till lock-on range is achieved.

The guidance scheme that the missile uses to guide itself towards the virtual target is the conventional PN law. Thus, the actual implementation of the VST guidance law has the same order of complexity as the conventional PN. But note that PN is used to guide the missile towards the virtual target and not towards the actual target. However, after lock-on in the homing phase the missile uses PN to guide itself towards the actual target.

\section{B. Sliding Speed}

One of the crucial factors in the VST guidance law is the determination of the sliding speed of the virtual target. This can be modeled as a linear constant speed slide or a nonlinear variable speed slide. We examine three possibilities here with reference to Fig. 3 where the distance between the virtual target position $P_{i}$ and the corresponding predicted intercept point PIP $i$ is denoted by $D_{i}$ at the beginning of a guidance cycle.

Linear Slide: The sliding speed of the virtual target during this guidance cycle is denoted by $v_{i l}$ and is given by

$$
v_{i l}=D_{i} / t_{\mathrm{go}}
$$

where $t_{\mathrm{go}}$ denotes the time-to-go. Note that if the PIP and $t_{\mathrm{go}}$ are accurate and thus invariant (as it happens in an ideal case), the sliding speed remains constant. In practice, the sliding speed may vary due to the target maneuver and the variation in $t_{\mathrm{go}}$ due to missile speed variation.

Nonlinear Initially Faster Slide: The sliding speed is denoted by $v_{\text {inlf }}$ and is given by

$$
v_{\text {inlf }}=\frac{D_{i} F e^{f t}}{t_{\mathrm{go}}}=v_{i l} F e^{f t}
$$

where $t$ denotes the elapsed time. The fast sliding parameters $F>0$ and $f<0$ are constants. Note that the sliding speed is initially high and subsequently drops to lower values as the virtual target approaches the PIP. This causes the missile to reach an altitude lower than that in the case of linear slide before the missile pitches down towards the PIP.

Nonlinear Initially Slower Slide: The sliding speed is denoted by $v_{i n l s}$ and is given by

$$
v_{i n l s}=\frac{D_{i} S\left(e^{s t}-1\right)}{t_{\mathrm{go}}}=v_{i l} S\left(e^{s t}-1\right)
$$

where the slow sliding parameters $S>0$ and $s>0$ are constants. The sliding speed is initially low and rises as the virtual target approaches the PIP. This causes the missile to reach an altitude higher than that in case of linear slide before pitching down.

Thus, the sliding of the virtual target, which in turn modulates the missile trajectory, can be controlled by selecting the position of the initial position $P_{0}$ of the virtual target and the sliding parameters $F$ and $f$ or $S$ and $s$. We later show in the simulations that selecting the location of $P_{0}$ and one of the parameters $f$ or $s$ is sufficient to exercise adequate control on the missile trajectory.

\section{Computation of $t_{\mathrm{go}}$ and PIP}

To determine the sliding velocity for the virtual target we need to compute $t_{\mathrm{go}}$ and the PIP. The PIP is not a fixed point for the entire engagement. It changes its position at every guidance cycle due to target maneuver and time-varying missile velocity and position. So at the beginning of every guidance cycle we need to compute the PIP. For this, from Fig. 3, we first compute $t_{\mathrm{go}}$ as

$$
t_{\mathrm{go}}=\frac{r}{V_{t} \cos \left(\alpha_{t}-\theta\right)-V_{m} \cos \left(\alpha_{m_{\text {ideal }}}-\theta\right)}
$$


where $V_{m}$ is the instantaneous velocity of the missile and $\alpha_{m_{\text {ideal }}}$ is calculated from the ideal collision course as follows: If

$$
\left|\left(V_{t} / V_{m}\right) \sin \left(\alpha_{t}-\theta\right)\right| \leq 1
$$

then,

$$
\alpha_{m_{\text {ideal }}}=\theta+\sin ^{-1}\left\{\left(V_{t} / V_{m}\right) \sin \left(\alpha_{t}-\theta\right)\right\}
$$

else

$$
\alpha_{m_{\text {ideal }}}=\alpha_{m} .
$$

Note that (5) represents the condition when the magnitude of the component of the target velocity normal to the line-of-sight (LOS) is less than the target speed. Satisfaction of this condition allows us to compute $t_{\mathrm{go}}$ from the ideal collision triangle geometry. The PIP is computed by projecting the current target position $\left(x_{t}, y_{t}\right)$ forward by $t_{\mathrm{go}}$. For example, if we assume a constant speed target then PIP $=\left(x_{t}+\right.$ $V_{t} t_{\mathrm{go}} \cos \alpha_{t}, y_{t}+V_{t} t_{\mathrm{go}} \sin \alpha_{t}$ ). If target acceleration is known then a suitable extrapolation can be carried out. Finally, although it may appear that a constant missile velocity is used for the time-to-go calculation, note that the value used is the instantaneous missile velocity obtained from the state equations and so, the variation in missile velocity is accounted for at every guidance cycle.

\section{SIMULATION}

\section{A. Models}

The propulsive and geometric characteristics of the missile depend mostly upon the range and average velocity for which we wish to design the guidance law. Some of the important missile propulsive and geometric parameters are given in Table I. We assume the thrust profile given in the Fig. 4. A solid propellant rocket with a boost-sustain configuration is used. The thrust generated is proportional to the rate of change of the propellant mass $\dot{m}$ during different time spans, and is assumed to be

$$
\begin{array}{ll}
\dot{m}=-8 \mathrm{~kg} / \mathrm{s}, & 0 \leq t \leq 5 \\
\dot{m}=-1 \mathrm{~kg} / \mathrm{s}, & 5<t \leq 40 \\
\dot{m}=0 \mathrm{~kg} / \mathrm{s}, & t>40 .
\end{array}
$$

Density and temperature of the atmosphere are functions of altitude. So, for a standard atmosphere, we use the following standard formulae [14]:

$$
\begin{aligned}
& \text { If } h \leq 11 \mathrm{~km} \\
& \qquad \begin{aligned}
\hat{T} & =288.16-0.0065 h^{\circ} \mathrm{K} \\
\rho & =1.225\left(\frac{\hat{T}}{288.16}\right)^{-((g / a R)+1)} \mathrm{kg} / \mathrm{m}^{3} .
\end{aligned}
\end{aligned}
$$

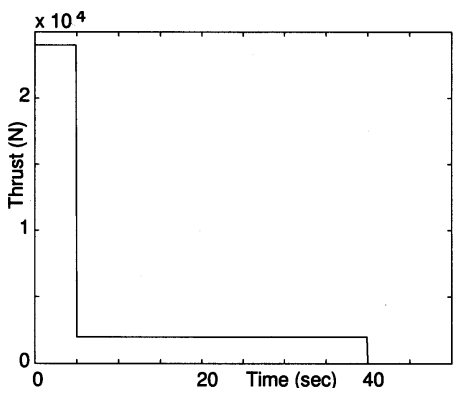

Fig. 4. Thrust profile.

TABLE I

Missile Specifications

\begin{tabular}{cc}
\hline \hline Parameter & Value \\
\hline Diameter of the Missile & $300 \mathrm{~mm}$ \\
Length of the Missile & $4000 \mathrm{~mm}$ \\
Mass of the Missile & $165 \mathrm{~kg}$ \\
Weight of Propellant & $75 \mathrm{~kg}$ \\
Burn Time & $40 \mathrm{~s}$ \\
\hline
\end{tabular}

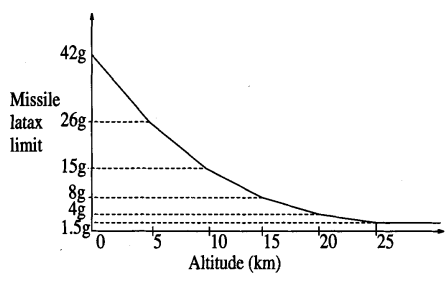

Fig. 5. Missile latax limits.

If $h>11 \mathrm{~km}$

$$
\begin{aligned}
\hat{T} & =216.66^{\circ} \mathrm{K} \\
\rho & =0.3655 e^{-(g(h-11000) / 288 \hat{T})} \mathrm{kg} / \mathrm{m}^{3} .
\end{aligned}
$$

where $h$ is the altitude in meters, $\hat{T}$ is the temperature in degrees Kelvin, $g$ is the acceleration due to gravity, $a$ is the lapse rate $(=-0.0065)$, and $R$ is the gas constant $(=288)$.

The missile is subject to lateral acceleration limits or load factor limits which vary with altitude and mainly arise due to structural limitations, control surface actuator limitations, and autopilot stability considerations. The load factor $\eta$ commanded by the guidance strategy should satisfy $|\eta| \leq \eta_{\max }$ where, $\eta=L / W$, and $L$ is the lift and $W$ is the weight of missile. This can be equivalently written as,

$$
\left|a_{m}\right| \leq \eta_{\max } g=a_{\max } .
$$

In the simulations the lateral acceleration limit $a_{\max }$ is expressed as a function of altitude and is assumed to have values shown in Fig. 5.

Fig. 6 shows the missile force balance. The missile is modeled as a point mass and the angle of attack $\alpha$ 


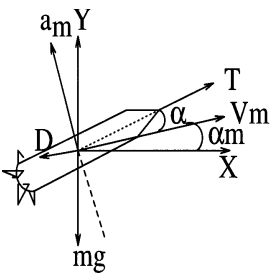

Fig. 6. Missile force balance.

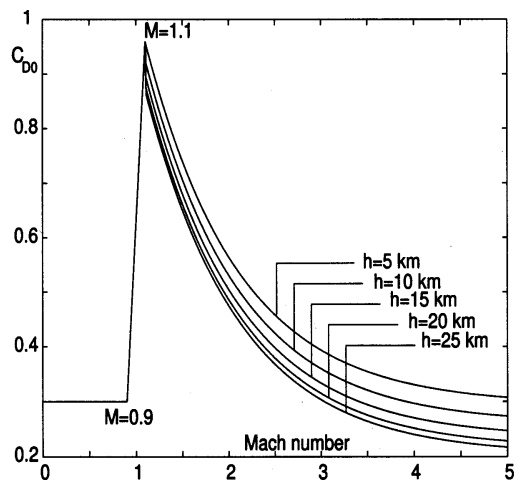

Fig. 7. $C_{D 0}$ profile.

is assumed to be zero. So the equations of motion are

$$
\begin{aligned}
\dot{V}_{m} & =\frac{T-D}{m}-g \sin \alpha_{m} \\
\dot{\alpha}_{m} & =\frac{a_{m}-g \cos \alpha_{m}}{V_{m}} \\
\dot{X}_{m} & =V_{m} \cos \alpha_{m} \\
\dot{Y}_{m} & =V_{m} \sin \alpha_{m}
\end{aligned}
$$

where

$$
\begin{aligned}
D & =\frac{1}{2} \rho V_{m}^{2} S C_{D} \\
C_{D} & =C_{D 0}+C_{D i}
\end{aligned}
$$

where $S$ is the wetted surface area, $C_{D 0}$ is the zero lift drag coefficient, and $C_{D i}$ is the induced drag coefficient. Note that although we assume the angle of attack to be zero in the equations of motion the non-zero induced drag coefficient takes care of the maneuver induced drag. The drag coefficients are functions of the Mach number $M$ which is, in turn, a function of $V_{m}$ and altitude $h$. The $C_{D 0}$ curve is shown in Fig. 7. The induced drag coefficient is given by

$$
C_{D i}=k C_{L}^{2}=k \frac{m^{2} a_{m}^{2}}{\left(\frac{1}{2} \rho V_{m}^{2} S\right)^{2}}
$$

where we assume $k=0.03$. This value of $k$ corresponds to a missile of fairly high aspect ratio. Note that the choice of $k$ depends on the missile

\begin{tabular}{|c|c|c|c|c|}
\hline $\begin{array}{c}\text { Guidance } \\
\text { Law }\end{array}$ & $\begin{array}{c}\text { Initial Position } \\
P_{0} \text { of Virtual } \\
\text { Target }(\mathrm{m})\end{array}$ & $\begin{array}{l}\text { Intercept } \\
\text { Time for } \\
\text { Receding } \\
\text { Target (m) }\end{array}$ & $\begin{array}{c}\text { Intercept } \\
\text { Time for } \\
\text { Approaching } \\
\text { Target(s) }\end{array}$ & $\begin{array}{c}\text { Transition } \\
\text { Speed for } \\
\text { Approaching } \\
\text { Target }(\mathrm{m} / \mathrm{s})\end{array}$ \\
\hline VST & $(1000,15000)$ & 97.7 & 60.8 & 310 \\
\hline VST & $(3000,15000)$ & 85.9 & 59.8 & 312 \\
\hline VST & $(5000,15000)$ & 78.2 & 59.1 & 313 \\
\hline VST & $(7000,15000)$ & 72.5 & 58.6 & 311 \\
\hline VST & $(5000,5000)$ & 43.6 & 48.5 & 312 \\
\hline VST & $(5000,10000)$ & 62.3 & 53.8 & 312 \\
\hline VST & $(5000,20000)$ & 91.5 & 64.2 & 312 \\
\hline PN & - & 44.0 & 57.2 & 268 \\
\hline
\end{tabular}
geometry and affects the performance of a missile using either optimal control, SP, or VST guidance laws as all these guidance laws try to exploit the drag variations on the missile at different altitudes.
TABLE II

Comparison of Intercept Times in VST and PN

\section{B. Engagement Trajectories}

The engagement scenario chosen to illustrate the guidance law performance has a nonmaneuvering target flying with constant velocity of $200 \mathrm{~m} / \mathrm{s}$. The initial missile flight path angle $\alpha_{m}$ is taken as $10 \mathrm{deg}$. It is assumed that the missile loses its specific energy when its speed falls below $240 \mathrm{~m} / \mathrm{s}(\cong 1.2$ times the target speed) and the missile is no longer capable of intercepting the target. This serves as a realistic termination condition for the engagement as compared with an artificial constant endurance time.

Below, we compare the trajectories, missile speed and latax, missile speed at transition to lock-on for approaching and receding targets, and interception times of the VST guidance law, using the virtual target sliding models given above, with corresponding values obtained from a conventional PN trajectory .

Linear Slide: Table II shows the interception times for both receding and approaching target. For the receding target, the target's initial position is $(4 \mathrm{~km}, 1 \mathrm{~km})$ and for the approaching target it is $(24 \mathrm{~km}, 1 \mathrm{~km})$. The midcourse phase continues till the missile is within $3 \mathrm{kms}$ of the target at which point lock-on is achieved and a transition to the terminal homing phase occurs. The initial position $P_{0}$ of the virtual target is varied to obtain different missile trajectories. The first set of data in Table II corresponds to a variation in the horizontal range of $P_{0}$ while keeping its altitude fixed at $15 \mathrm{kms}$ and the second set of data corresponds to variation in the altitude of $P_{0}$ while keeping its horizontal range fixed at $5 \mathrm{kms}$.

In the receding target case, when the initial position of the virtual target $P_{0}$ is at a high altitude, the interception time for VST guidance law is substantially higher than the PN law. This situation improves marginally as the horizontal range of $P_{0}$ increases. The main reason behind this phenomena is that the missile flies through an unnecessarily longer path which is caused by the necessity to rise to very 
high altitudes. A substantial amount of time is wasted during the initial stages, when the missile speed is low, to achieve the high altitude demanded by the VST guidance law. Also, due to the pitch-up and pitch-down maneuvers, the latax pulled by the missile is high and the corresponding maneuver-induced drag is also high at the initial stages. The only observable advantage is that the VST guidance law gives a higher transition speed at lock-on and, presumably, a larger launch envelope.

On the other hand, when the target is an approaching one, the situation improves quite substantially and the interception times of VST, though still higher, are comparable to that of PN (with the maximum increase being about $6 \%$ over the PN value). The missile speed at the point of transition to lock-on is still higher (by about 16\%) than the corresponding PN value. As we see later, the corresponding launch envelope is fairly large for the VST guidance law due to this reason.

In the second set of data given in Table II, the horizontal range of the initial position of the virtual target $P_{0}$ is kept constant at $5 \mathrm{kms}$ while its altitude is varied from $5 \mathrm{kms}$ to $20 \mathrm{kms}$. Even here, for the receding target case, the best interception time is obtained when the position of $P_{0}$ is $(5 \mathrm{~km}, 5 \mathrm{~km})$. As its altitude increases, the interception time also increases. The reason for this is the same as explained earlier for the first data set. However, there is a significant improvement in interception time for the approaching target case. The interception time is about $15 \%$ lower than the corresponding PN value when the $P_{0}$ position is $(5 \mathrm{~km}, 5 \mathrm{~km})$ and about $6 \%$ lower when the $P_{0}$ position is $(5 \mathrm{~km}, 10 \mathrm{~km})$. However, when the $P_{0}$ altitude is higher than $10 \mathrm{kms}$, the interception time performance shows sharp degradation. In all these cases the missile speed at transition to lock-on is $16 \%$ higher for VST over the corresponding PN value.

The engagement trajectories, and velocity and acceleration profiles are shown in Figs. 8, 9, 10, and 11 for all the above cases. These trajectories show that in a linear sliding scheme we can control the missile trajectory by selecting the initial position of the virtual target. Also the missile speed curves show the higher speeds attained by the VST guidance when compared with PN guidance. However, due to the shape of the VST trajectories the maneuver levels are higher for VST than for PN. Further, from these results we can reasonably conclude that a careful choice of the initial virtual target position can improve the performance of the empirical VST guidance law with the linear target sliding model quite significantly in terms of interception time as well as higher energy during the terminal phase.

Nonlinear Initially Faster Slide: Here we consider only the approaching target case which yields a significant performance improvement over PN. Fig. 12 shows the variation against time of the sliding speed
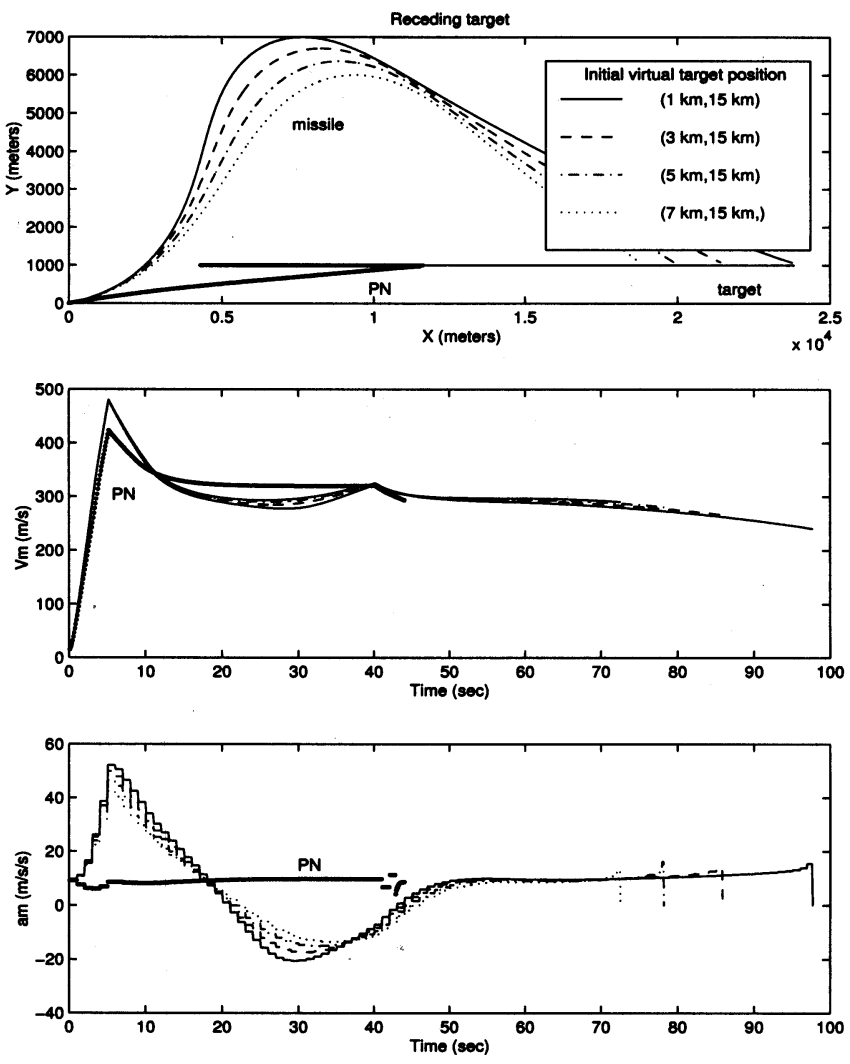

Fig. 8. Trajectories for receding target with variation in horizontal range of initial virtual target position.
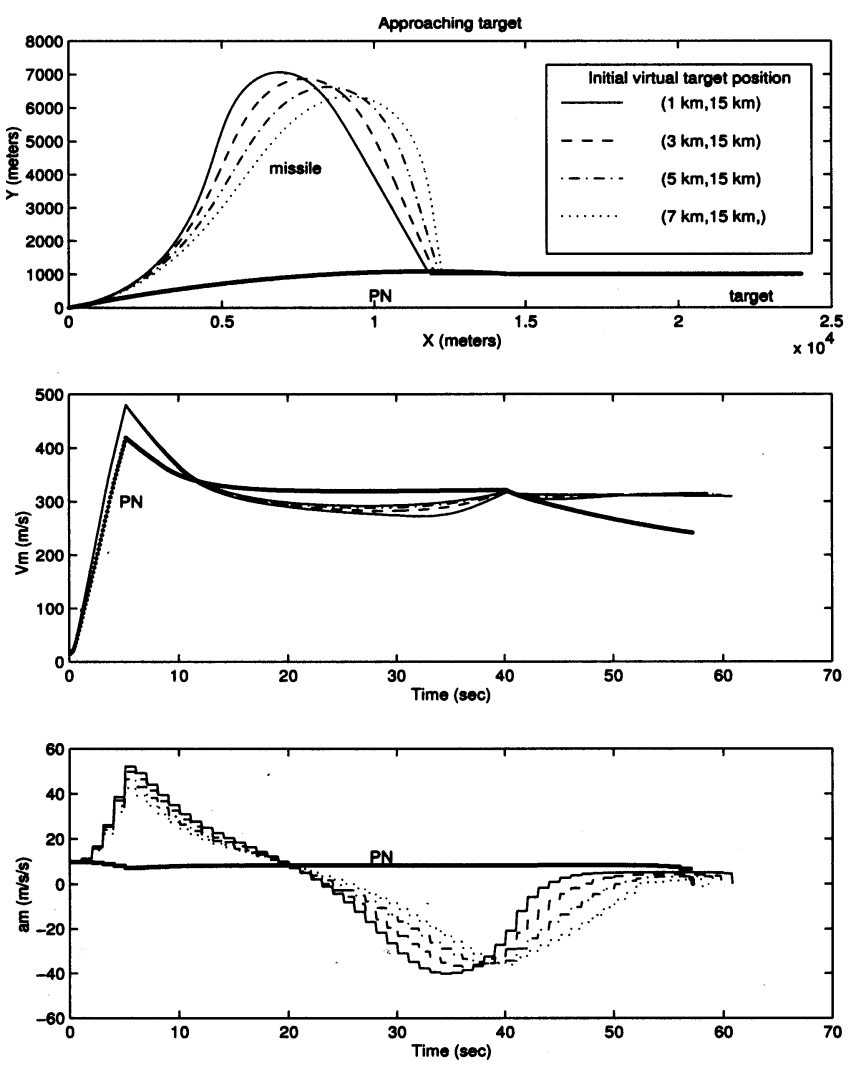

Fig. 9. Trajectories for approaching target with variation in horizontal range of initial virtual target position. 

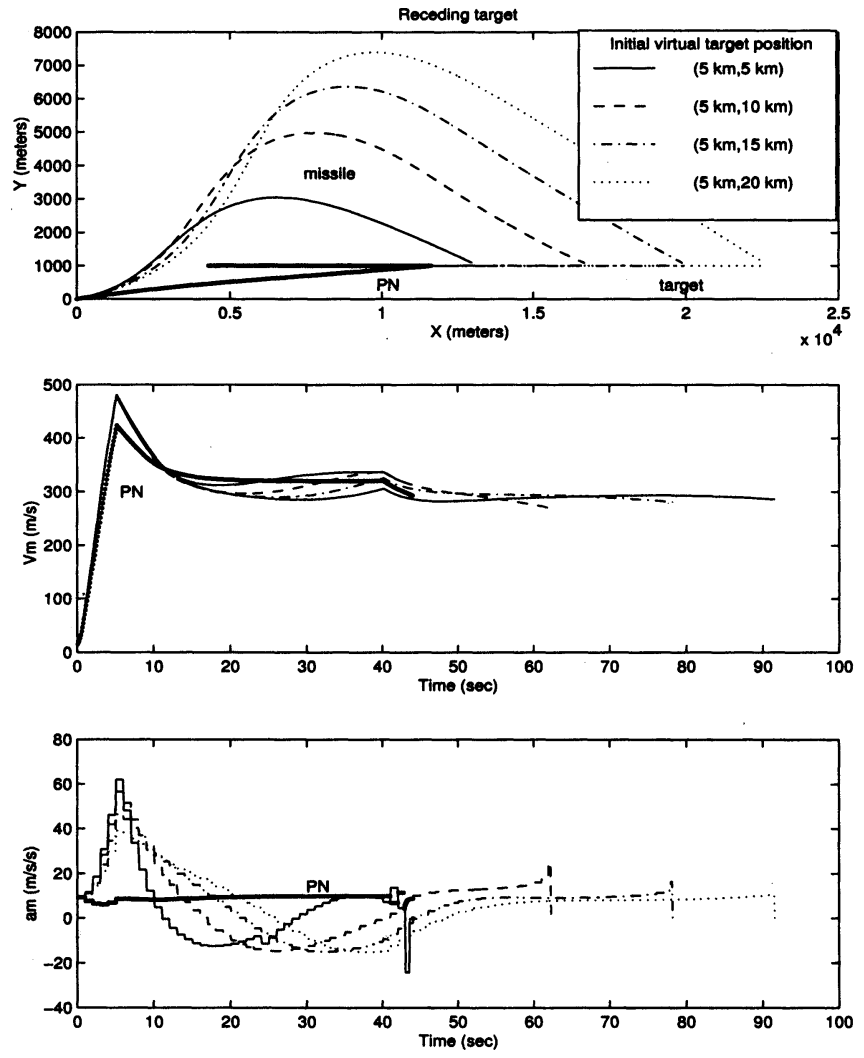

Fig. 10. Trajectories for receding target with variation in altitude of initial virtual target position.
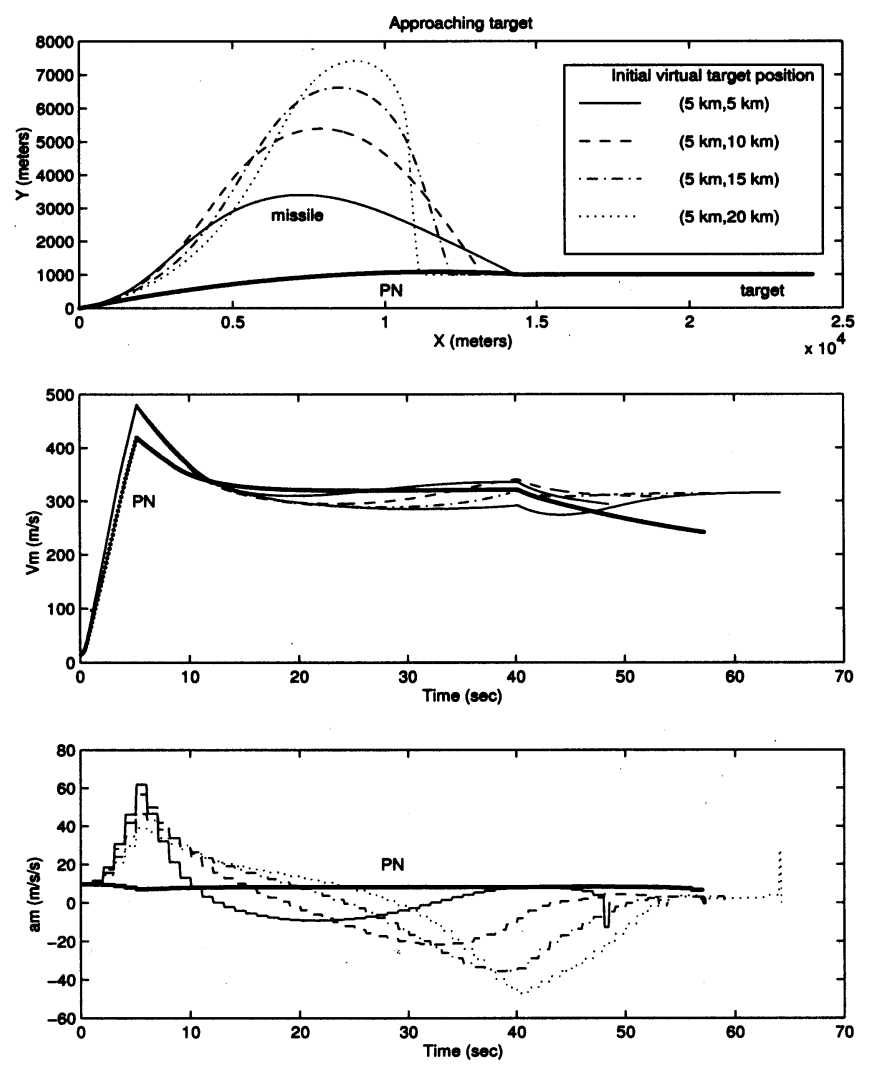

Fig. 11. Trajectories for approaching target with variation in altitude of initial virtual target position.

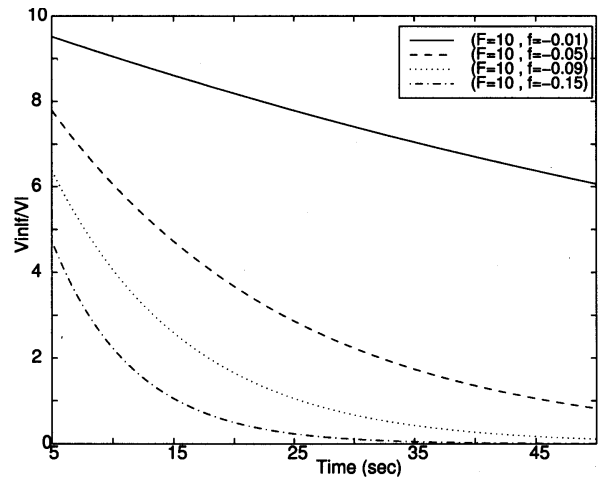

Fig. 12. Sliding speed of virtual target for initially faster slide.

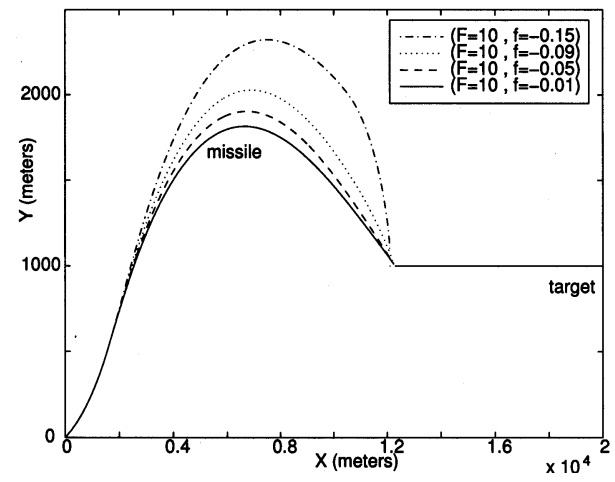

Fig. 13. Trajectories for initially faster sliding.

normalized with respect to the linear sliding speed for different values of $F$ and $f$. From this figure it may appear that since the sliding speed of the virtual target converges towards zero with time (especially for high negative values of $f$ ), the virtual target will stop chasing a receding target. But this situation will never occur since the sliding speed of the virtual target is proportional to the distance between the virtual target position and the PIP. So, as long as the virtual target is at a definite distance from the PIP, it would have a substantial sliding speed. Moreover, the fact that this scheme is used only during midcourse, which ends when the missile is at the target lock-on range (which has a fairly high value), ensures that the sliding speed never actually becomes zero. The intention of the exponential term is to ensure that the sliding speed is high initially and then tapers off gradually towards the end of the midcourse phase. We consider four different values of $f$. The sliding speed is fairly large initially and reduces exponentially as the virtual target approaches the PIP. Since the virtual target loses altitude rapidly in the initial stages, the missile does not attain very high altitudes.

Fig. 13 shows the missile and target trajectories for the four cases. Note that the downrange and altitude scales have been chosen differently in order to show the different trajectories, and so the actual trajectory is less humped than that shown in the figure. The initial location $P_{0}$ of the virtual target is at $(5 \mathrm{~km}, 5 \mathrm{~km})$. 


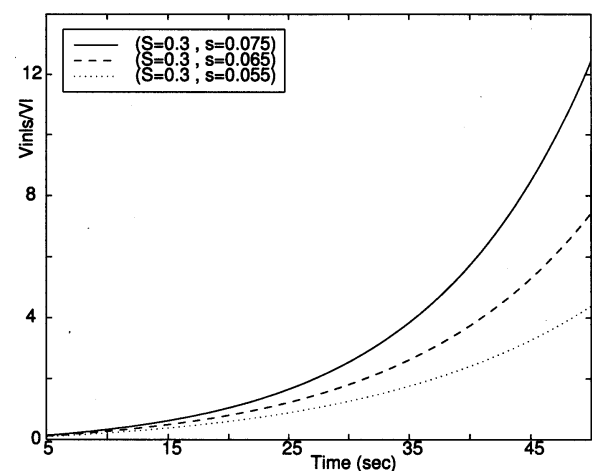

Fig. 14. Sliding speed of virtual target for initially slower slide.

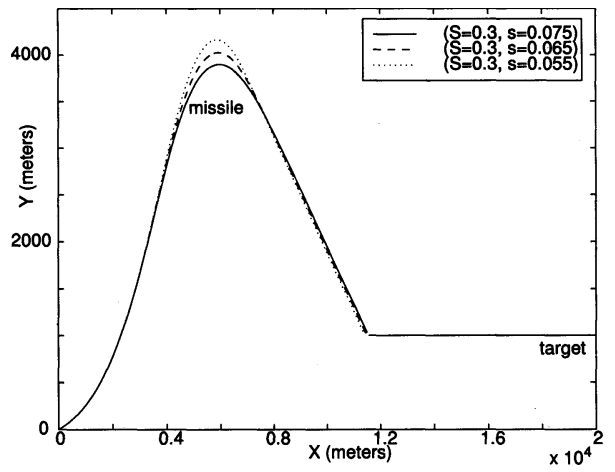

Fig. 15. Trajectory comparison for initially slower sliding.

With the change in the value of the sliding parameter $f$, the maximum altitude to which the missile rises also varies. However, the interception time does not vary much in the four cases and is about $40 \mathrm{~s}$. So, it appears that the interception time is not sensitive to the variation in the sliding parameters as long as the position of $P_{0}$ is kept fixed.

Nonlinear Initially Slower Slide: Here too, we consider only an approaching target. Fig. 14 shows the variation against time of the sliding speed normalized with respect to the linear sliding speed for different values of $S$ and $s$. We consider four different values of $s$. The sliding speed is low initially and increases exponentially as the virtual target approaches the PIP. Since the virtual target loses altitude slowly in the initial stages, the missile attains high altitudes.

Fig. 15 shows the missile and target trajectories for the three cases. As before, the scales for altitude and downrange are different and $P_{0}$ is at $(5 \mathrm{~km}, 5 \mathrm{~km})$. Here too the choice of the sliding parameters affects the maximum altitude to which the missile rises. The altitude reached by the missile is considerably higher than in the previous case. The interception time is also higher (about $42 \mathrm{~s}$ ) but does not vary much in the three cases. Thus, even here the interception time is not very sensitive to the variation in the sliding parameters so long as the position of $P_{0}$ is kept fixed.

Comparison of Trajectories: To compare the performance of these three sliding schemes against PN we select a specific engagement geometry with a

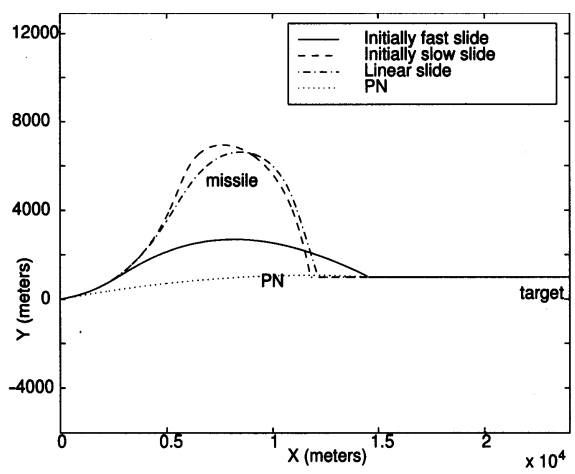

Fig. 16. Comparison of trajectories.

TABLE III

Comparison of Interception Times

\begin{tabular}{ccc}
\hline \hline Guidance Law & Sliding Scheme & Interception Time (s) \\
\hline VST & Linear & 59.14 \\
& Initially slow & 60.93 \\
& Initially fast & 47.26 \\
\hline PN & - & 57.21 \\
\hline
\end{tabular}

nonmaneuvering approaching target. Fig. 16 shows the trajectories obtained with the best combination of the sliding parameters and the position of $P_{0}$ with respect to interception time. Interception time achieved is considerably less for the nonlinear initially faster sliding case, compared with PN, the linear slide case, and the nonlinear initially slow sliding case. Table III gives the interception times for the above cases. This example shows that by selecting the sliding parameters and the initial position of the virtual target we can improve upon the interception time. This result also shows that the altitude reached by the missile during the pitch-up maneuver plays a crucial role in the interception time performance.

\section{Launch Boundary Envelopes}

One of the very effective performance measures for a guidance law is its launch envelope in engaging approaching and receding targets. In fact, it is a significant factor when we consider the performance of a guidance law implemented in the midcourse phase. The upper launch boundary generally constitutes of the farthest points at which the target may be initially located and still be intercepted by the missile. The upper launch boundary is mainly caused by the limit on the flight time of the missile, or on the rate at which its speed drops after the sustained-thrust phase is over. Similarly, the lower launch boundary constitutes of the closest initial target positions at which the interception is possible. The lower boundary is mainly caused by the constraints on the missile velocity profile, lateral acceleration limits, and guidance initiation time. 


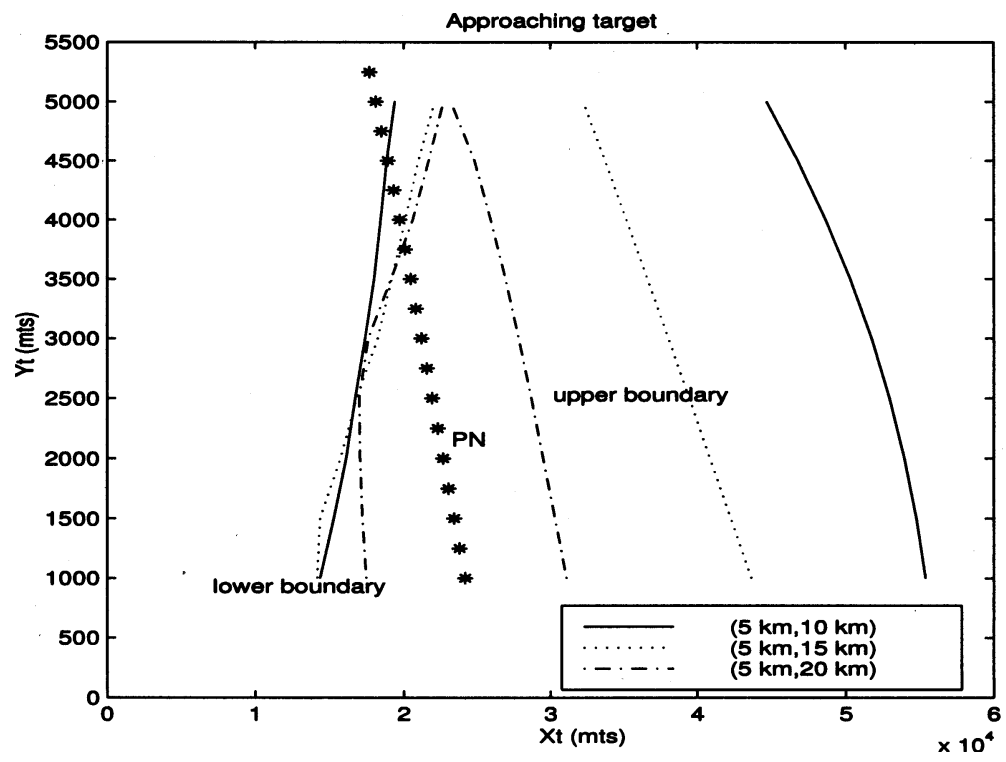

Fig. 17. Launch boundaries for approaching targets with variation in altitude of initial virtual target position.

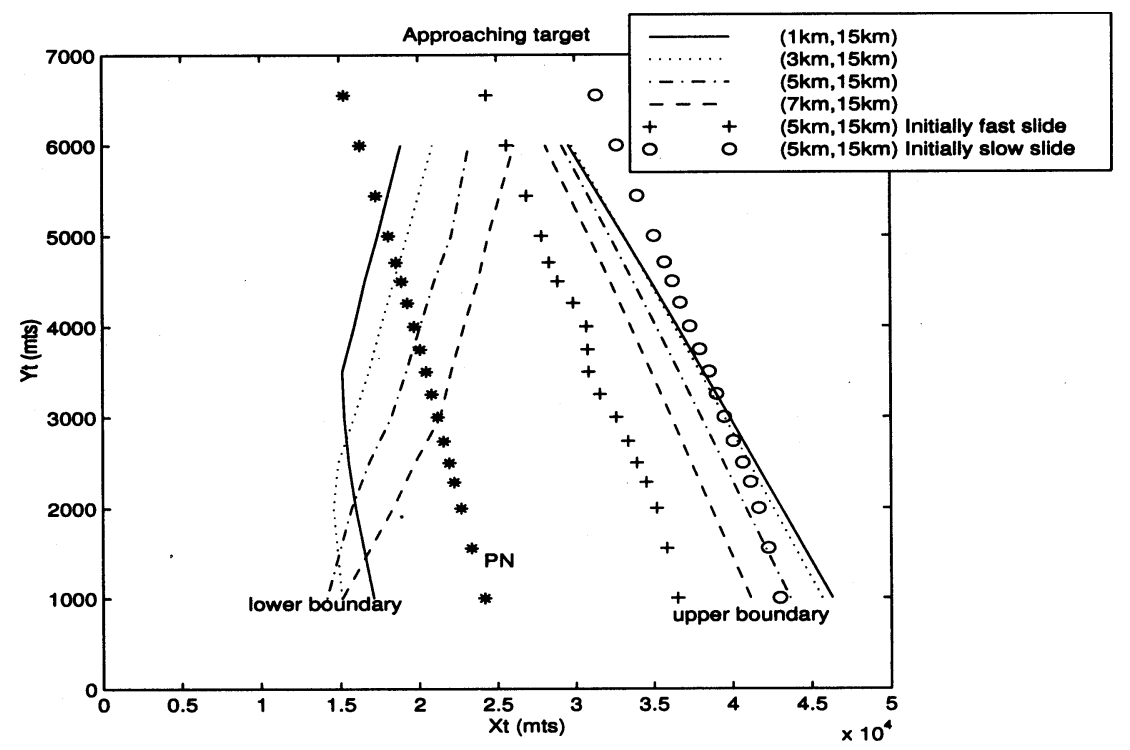

Fig. 18. Launch boundaries for approaching targets with variation in horizontal range of initial virtual target position.

Launch boundaries may be obtained for the worst case target behavior (that is, for a maneuvering target that attempts to evade the missile using optimal escape maneuvers) or for a specified target behavior (that is, nonmaneuvering targets or targets that maneuver in a fixed predetermined way). However, the first step in carrying out a performance evaluation using launch boundary envelopes almost always involves an approaching level target that flies at a constant altitude and speed. This is the simplest case and forms some sort of a benchmark since any guidance law that does not demonstrate good launch performance against this class of targets is unlikely to demonstrate good performance against other types of target behaviors. Therefore, for this simulation study, we also consider such a constant speed $(200 \mathrm{~m} / \mathrm{s})$ level flying target. A point to note is that the size of the launch boundary envelopes will change if higher target speeds are assumed. For instance, VST and PN both will have larger launch envelopes for approaching targets. But as long as we consider same target behavior, the relative positions of the launch boundaries of $\mathrm{PN}$ and VST will remain more or less the same.

The envelopes obtained are shown in Figs. 17 and 18. In Fig. 17 only linear sliding is considered with variation in altitude of the initial virtual target position $P_{0}$ and with a fixed horizontal range of $5 \mathrm{~km}$. Both the lower and upper boundaries have been obtained for the VST guidance law for approaching targets.

Fig. 18 shows the launch envelopes for linear sliding with variation in the horizontal range of the 
initial virtual target position for approaching targets, with a fixed altitude of $15 \mathrm{~km}$. This figure also gives the upper launch envelope boundaries for initially fast sliding case and initially slow sliding case.

These results clearly show the superiority of VST over PN in terms of extending the launch boundaries. These results also show that the choice of the sliding parameters and the initial virtual target position can help in extending the launch boundary considerably. The initially fast sliding scheme appears to be advantageous for receding targets whereas initially slow sliding scheme is advantageous for approaching targets.

\section{CONCLUSIONS}

In this paper we exploited the fact that at higher altitudes the low atmospheric density results in a lower drag on the missile to design an empirical VST guidance law. The resulting guidance law is simple to implement since it uses the conventional $\mathrm{PN}$ as its basic guidance strategy while at the same time it exploits the aerodynamic characteristics of the missile to deliver superior performance. These studies reveal that the selection of the sliding parameters and the initial position of the virtual target affect the achieved missile altitude and are important design considerations. As shown in this paper, it is possible to select reasonable values for these parameters based on intuition and experience. However, optimal performance requires a careful selection of these parameters. The issue of exact optimality is not explicitly addressed here as it would require very extensive simulations to be carried out for both nonmaneuvering and maneuvering targets. However, this would certainly be a useful direction of research that would serve to put the VST guidance law on a firmer footing. Some preliminary results are available in [15] on the optimization of these guidance parameters. However, the presentation of these results would require a separate treatment and is beyond the scope of the present paper. Another point worth noting is that although the PN guidance law has been used to generate the missile latax, there is no constraint in using only the PN law. One can use any other guidance law in its place (depending on how much complexity one can incorporate) and get similar benefits so long as the artifice of the virtual target is used for missile trajectory shaping. The main objective of this paper was to draw attention to the fact that the optimal performance obtained from optimal control based guidance laws formulated on a complex missile-target engagement model can be emulated to a large extent by empirical guidance laws that exploit the physics of the engagement scenario.

\section{REFERENCES}

[1] Yang, C-D., and Yang, C-C. (1996) Analytical solution of 3D true proportional navigation. IEEE Transactions on Aerospace and Electronic Systems, 32, 4 (Oct. 1996), 1509-1522.

[2] Duflos, E., Penel, P., and Vanheeghe, P. (1999) $3 \mathrm{D}$ guidance law modelling. IEEE Transaction on Aerospace and Electronic Systems, 35, 1 (Jan. 1999), 72-83.

[3] Ghose, D. (1994)

On the generalization of true proportional navigation. IEEE Transaction on Aerospace and Electronic Systems, 30, 2 (Apr. 1994), 545-555.

[4] Leng, G. (1998)

Guidance algorithm design: A nonlinear inverse approach. Journal of Guidance, Control, and Dynamics, 21, 5 (Sept.-Oct. 1998), 742-746.

[5] Yang, C-D., and Yang, C-C. (1997) Optimal pure proportional navigation for maneuvering targets.

IEEE Transaction on Aerospace and Electronic Systems, 33, 3 (July 1997), 949-957.

[6] Pfleghaar, W. (1985)

The general class of optimal proportional navigation. Journal of Guidance, Control, and Dynamics, 8, 1 (Jan.-Feb. 1985), 144-147.

[7] Sridhar, B., and Gupta, N. K. (1980)

Missile guidance laws based on singular perturbation methodology.

Journal of Guidance and Control, 3, 2 (Mar.-Apr. 1980), 158-165.

[8] Cheng, V. H. L., and Gupta, N. K. (1986)

Advance midcourse guidance for air-to-air missiles. Journal Guidance, Control, and Dynamics, 9, 2 (Mar.-Apr. 1986), 135-142.

[9] Bharadwaj, S., Wu, M., and Mease, K. D. (1997)

Identifying time-scale structure for simplified guidance law development.

In Proceedings of the AIAA Guidance, Navigation and Control Conference, New Orleans, LA, Aug. 1997.

[10] Bharadwaj, S., and Mease, K. D. (1999)

Geometric structure of multiple time-scale nonlinear systems.

In Proceedings of the IFAC World Congress, Beijing, July 1999.

[11] Mease, K. D., Iravanchy, S., Bharadwaj, S., and Fedi, M. (2000)

Time scale analysis for nonlinear systems. In Proceedings of the AIAA Guidance, Navigation, and Control Conference, Denver, CO, Aug. 2000, paper 2000-4591.

[12] Imado, F., Kuroda, T., and Miwa, S. (1990)

Optimal midcourse guidance for medium-range air-to-air missiles.

Journal of Guidance, Control, and Dynamics, 13, 4 (July-Aug. 1990), 603-608.

[13] Raikwar, A. G. (1997)

A midcourse guidance law for a BVRAAM using singular perturbation technique.

Master of Engineering project report, Dept. of Aerospace Engineering, Indian Institute of Science, Bangalore, India, Jan. 1997.

[14] Anderson, J. D. (1989)

Introduction to Flight (3rd ed.).

New York: McGraw-Hill, 1989.

[15] Sharma, M. K., and Ghose, D. (2002)

Optimization of a BVRSAM trajectory using the virtual sliding target guidance law.

Technical report G\&C/DG/001/02, Dept. of Aerospace Engineering, Indian Institute of Science, Bangalore, India, Aug. 2002. 

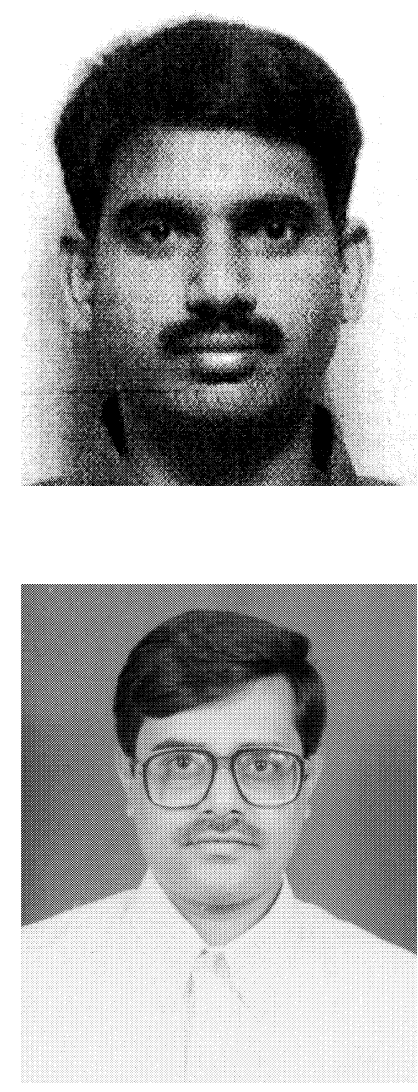

P. A. Raju obtained a B.E. degree in electronics and communication engineering from Andhra University, Visakhapatnam, India, in 1996, and an M.E. degree in aerospace engineering from the Indian Institute of Science, Bangalore, in 1999.

$\mathrm{He}$ is presently engaged in developing telecommunication software for NORTEL Networks, Dallas, TX.

Debasish Ghose (M'03) obtained a B.Sc. (Engg) degree from the National Institute of Technology (formerly known as the Regional Engineering College), Rourkela, India, in 1982, and an M.E. and a Ph.D. degree, from the Indian Institute of Science, Bangalore, in 1984 and 1990, respectively.

$\mathrm{He}$ is presently an associate professor of Aerospace Engineering at the Indian Institute of Science. His research interests are in guidance and control of aerospace vehicles, distributed decision-making systems, and scheduling problems in distributed computing systems. He has held visiting positions at several other universities such as the University of California at Los Angeles and the Kangwon National University in South Korea. He was also selected for the Alexander von Humboldt fellowship for the year 2000. 